\title{
Controlling nutritional status score as a prognostic marker to predict overall survival in resected biliary tract cancers
}

\author{
Lejia Sun ${ }^{1 \#}$, Si Su ${ }^{2 \#}$, Jianping Xiong ${ }^{3 \#}$, Wenmo $\mathrm{Hu}^{2}$, Lei Liu ${ }^{2}$, Haifeng $\mathrm{Xu}^{1}$, Shunda Du ${ }^{1}$, Haitao Zhao ${ }^{1}$, \\ Xin Lu ${ }^{1}$, Xinting Sang ${ }^{1}$, Shouxian Zhong ${ }^{1}$, Huayu Yang ${ }^{1}$, Yilei Mao ${ }^{1 \wedge}$ \\ ${ }^{1}$ Department of Liver Surgery, Peking Union Medical College (PUMC) Hospital, PUMC \& Chinese Academy of Medical Sciences, Beijing, China; \\ ${ }^{2}$ Peking Union Medical College (PUMC), PUMC \& Chinese Academy of Medical Sciences, Beijing, China; ${ }^{3}$ Interventional Radiology, Beijing \\ Friendship Hospital, Capital Medical University, Beijing, China \\ Contributions: (I) Conception and design: Y Mao, H Yang; (II) Administrative support: Y Mao, H Yang; (III) Provision of study materials or patients: \\ H Xu, S Du, H Zhao, X Lu, X Sang, S Zhong; (IV) Collection and assembly of data: W Hu, L Liu; (V) Data analysis and interpretation: L Sun, S Su, \\ J Xiong; (VI) Manuscript writing: All authors; (VII) Final approval of manuscript: All authors. \\ \#These authors contributed equally to this work. \\ Correspondence to: Yilei Mao. Department of Liver Surgery, Peking Union Medical College (PUMC) Hospital, PUMC \& Chinese Academy of \\ Medical Sciences, Beijing 100730, China. Email: pumch-liver@hotmail.com; Huayu Yang. Department of Liver Surgery, Peking Union Medical \\ College (PUMC) Hospital, PUMC \& Chinese Academy of Medical Sciences, Beijing 100730, China. Email: dolphinyahy@hotmail.com.
}

Background: The aim of our study was to explore the prognostic significance of the preoperative controlling nutritional status (CONUT) score and establish a nomogram to predict overall survival (OS) and to achieve a more accurate prognostic risk stratification.

Methods: Clinicopathological records of 371 patients who underwent surgical resection for biliary tract cancers (BTC) from December 2002 to December 2017 were reviewed retrospectively. The associations of the CONUT score with clinicopathological factors and OS were evaluated. Univariate and multivariable Cox regression analysis were used to screen out independent predictors. A nomogram was developed and validated to estimate OS.

Results: The CONUT score was an independent predictor of OS [hazard ratio $1.478,95 \%$ confidence interval (CI), 1.078-2.025, $\mathrm{P}=0.015]$. And patients with a high CONUT score tended to have a poor prognosis with poor differentiation $(\mathrm{P}=0.011)$ of tumor cells and longer hospital stays $(\mathrm{P}=0.046)$. Besides the CONUT score, carbohydrate antigen 19-9, surgical method, and the American Joint Committee on Cancer (AJCC; 7th edition) TNM stage were contained in the final prognostic model. An OS nomogram was generated to visually predict $1-, 3-$, and 5 -year OS. The C-index was 0.714 (95\% CI, 0.673-0.755) and 0.679 (95\% CI, 0.616-0.742) in the development and validation cohort respectively. The nomogram provided superior discriminative power than the AJCC TNM staging system. The nomogram also demonstrated good risk stratification power in the entire cohort of BTC patients as well as for both BTC and surgical method subgroups.

Conclusions: The nomogram based on the CONUT score can predict OS in patients with BTCs, and it performed better than the AJCC TNM staging system.

Keywords: Controlling nutritional status score (CONUT score); biliary tract cancer (BTC); prognosis; biomarker; nomogram

Submitted Oct 09, 2020. Accepted for publication Jan 12, 2021.

doi: $10.21037 /$ atm-20-6770

View this article at: http://dx.doi.org/10.21037/atm-20-6770

$\wedge$ ORCID: 0000-0003-0449-4223. 


\section{Introduction}

Biliary tract cancers (BTCs) include gallbladder cancer (GBC), intrahepatic (ICC) and extrahepatic (ECC) cholangiocarcinomas (1). BTCs, which are relatively rare globally, are associated with a very poor prognosis. Approximately 220,000 new BTC diagnoses and 165,000 BTC deaths worldwide were reported in $2018(2,3)$. There has been an increase in the incidence of BTCs, especially ICCs (4-6). It has been broadly agreed that surgery is the only potentially curative treatment for patients with BTC (7). However, there is a high recurrence rate even with complete resection, and the prognosis continues to be unsatisfactory, with a 5-year survival rate of approximately $18 \%$ in the US and $10 \%$ in Chile (2). Therefore, a better treatment strategy is needed to tackle this problem. Prognostic markers can be used to achieve accurate risk stratification and predict which patients would most likely benefit from surgery and to help guide the selection of the best treatment; this approach would have a great value for BTC patients.

The American Joint Committee on Cancer (AJCC; 7th edition) TNM staging system is the most widely used staging system to determine prognosis and treatment. However, this system considers only the depth of the primary tumor invasion, regional lymph node metastasis, and distant metastasis but not tumor heterogeneity and the patient's condition (8). Thus far, a few studies have reported reliable biomarkers that predict prognosis in patients, including carbohydrate antigen 19-9 (CA19-9), which is one of the major predictive factors for poor prognosis after surgery (9). In addition to traditional tumor biomarkers, cancer-related inflammatory and nutritional states are gaining increasing attention. The prognostic nutritional index (PNI) and inflammatory factors such as the preoperative neutrophil-to-lymphocyte ratio (NLR) and the platelet-to-lymphocyte ratio (PLR) have been reported to be useful biomarkers for predicting prognosis in many types of cancers, including GBC and cholangiocarcinomas (10-12). A recently developed novel and more comprehensive biomarker, namely, the controlling nutritional status (CONUT) score, has been widely used to indicate the nutritional and inflammatory states of patients. The score is calculated based on serum albumin levels, total cholesterol levels, and total lymphocyte count in peripheral blood (13). In addition, the preoperative CONUT score is reported as an independent prognostic marker in patients with various malignancies, including gastric cancer (14), esophageal cancer (15), colorectal cancer (16), hepatocellular carcinomas (17), cholangiocarcinomas (18), lung cancer (19), and breast cancer (20). However, the prognostic significance of the CONUT score in patients with BTCs remains undetermined.

In this study, we explored the prognostic significance of the preoperative CONUT score and further established a nomogram to predict the overall survival (OS) of patients with BTCs to acquire more accurate prognostic risk stratification information and guide individualized choice of treatment. Our study is in accordance with the STROBE reporting checklist (available at http://dx.doi.org/10.21037/ atm-20-6770).

\section{Methods}

\section{Patients}

The clinicopathological records of 601 patients who underwent surgical resection for BTCs at the Peking Union Medical College Hospital from December 2002 to December 2017 were reviewed retrospectively. All enrolled patients met the following inclusion criteria: presence of pathologically confirmed GBC, ICC, or ECC detected using biopsy; undergoing surgical resection; no other primary malignant tumors; and complete data in clinicopathological records.

\section{Data collection}

Data of the following variables were collected and were analyzed: age, sex, with or without conditions such as diabetes mellitus (DM), hypertension (HT) and jaundice, CA19-9, differentiation of tumor cells, tumor size, lymphovascular invasion, perineural invasion, lymph node metastasis, TNM stage according to AJCC, surgical method including curative resection with clear margins (R0 resection) or not, complications according to the ClavienDindo classification (Clavien-Dindo $\geq \mathrm{II}$ or not), and length of hospital stay after surgery. In our study, continuous variables were transformed into categorical variables according to clinical significance and findings.

Preoperative CONUT scores were calculated using data of serum albumin concentration, total lymphocyte count, and cholesterol concentration collected from clinical records, and the computing method is shown in Table S1 (21). Patients were divided into the low and high groups based on the CONUT score: score of $\leq 1$, and $\geq 2$. 
OS was defined as the time from surgery to the date of death or last follow-up.

\section{Ethics statement}

The study was approved by the Clinical Research Ethics Committee of Peking Union Medical College Hospital (approval No. S-K1110). Informed consent forms were signed by all patients before surgery to permit the use of their clinical data in the study. All procedures performed in studies involving human participants were in accordance with the 1964 Helsinki Declaration and its later amendments or comparable ethical standards.

\section{Statistical analysis}

Continuous data were presented as the mean and standard deviation (SD) if normally distributed and the median and interquartile range (IQR) if not. Categorical variables are presented as numbers and percentages. Group comparisons were analyzed using the Chi square test and Wilcoxon sum rank test. Survival curves were obtained using the KaplanMeier method and compared according to the log-rank test. Variables that were significant on univariate analysis were entered into the multivariable Cox regression model, and a backward step selection was used to eliminate nonsignificant variables using a $\mathrm{P}$ value $<0.05$.

Patients were randomly allocated to a development cohort and a validation cohort at a ratio of $7: 3$. The nomogram was designed to predict 1-, 3-, and 5-year OS based on the development cohort according to the significant variables from the multivariable analysis. The performance of the nomogram was tested using Harrell's C-index (C-index), ROC curves and area under the curves (AUCs), calibration curves, and decision curve analysis (DCA) in both cohorts. Each patient was given a total point calculated according to the regression coefficient from the established Cox regression model of the development cohort. According to the quartile of total points, patients were divided into four risk groups: low risk, medium risk, high risk, and very high risk. Survival curves were depicted using the Kaplan-Meier method in the subgroups of interest, and the differences were tested using the log-rank test. SPSS 25.0 software and $\mathrm{R}$ project version 3.6.2 were used to perform the statistical analyses. All tests were twosided, and results with a $\mathrm{P}$ value $<0.05$ were considered statistically significant.

\section{Results}

\section{Basic characteristics}

Among the 601 reviewed cases, 230 with missing variables were excluded. The median follow-up time was 16 months. Of the 371 eligible patients, 172 (46.4\%) were female and 199 (53.6\%) were male, with ages ranging from 23 to 88 years $(61.5 \pm 10.4$ years). For the different types of BTC, there were 96 (25.9\%) GBC, 69 (18.6\%) ICC, and 206 (55.5\%) ECC cases. Regarding AJCC TNM stage, 117 patients $(31.5 \%)$ were stage $0-\mathrm{I}, 115(31.0 \%)$ were stage II, $107(28.8 \%)$ were stage III, and $32(8.6 \%)$ were stage IV. In total, $222(59.8 \%)$ patients received curative resection with clear margins, and the other 149 (40.2\%) did not. Detailed clinicopathological characteristics are presented in Table 1. Most stage IV patients underwent surgery because of a vague preoperative diagnosis. In these cases, 5 patients were found with distant metastases in the exploratory laparotomy and underwent palliative surgery, and in other 27 patients, only 8 patients obtained curative resection while the other 19 did not.

\section{Correlations between the CONUT score and clinicopathological characteristics}

Of the 371 BTC patients included in the study, 201 $(54.2 \%)$ were grouped in the low CONUT score group (CONUT $\leq 1)$ and 170 were grouped in the high CONUT score group (CONUT $\geq 2$ ). Patient clinicopathological characteristics stratified in the CONUT score group are shown in Table 1. High CONUT scores were correlated with poor differentiation $(\mathrm{P}=0.011)$ of tumor cells and longer hospital stays $(\mathrm{P}=0.046)$, which indicates that patients with high CONUT scores tended to have a poor prognosis. However, the CONUT score was not associated with age, sex, DM, HT, jaundice, preoperative CA19-9 level, tumor size, lymphovascular invasion, perineural invasion, lymph node metastasis, TNM stage, surgical method, or severe complications (all $\mathrm{P}>0.05$ ).

\section{Correlations between the CONUT score and OS}

The 1-, 3-, and 5-year OS rates were $64.9 \%, 29.3 \%$, and $20.5 \%$, respectively, in the high CONUT score group and $70.2 \%, 39.7 \%$, and $29.3 \%$, respectively, in the low CONUT score group. According to Kaplan-Meier survival analysis, patients with high CONUT scores were significantly 
Page 4 of 14

Sun et al. The prognostic value of CONUT in resected BTC

Table 1 Basic clinicopathological characteristics by CONUT score group

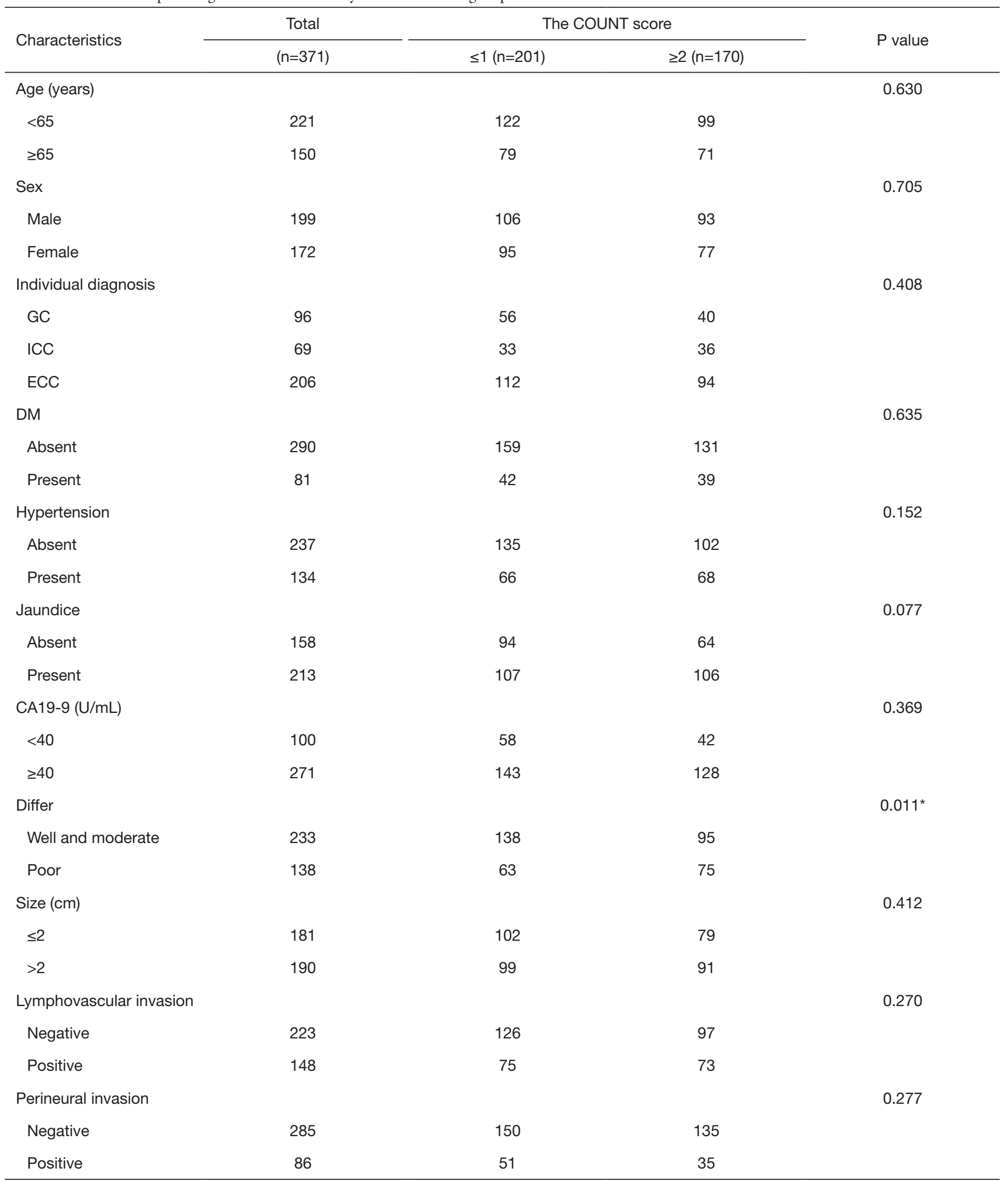

Table 1 (continued) 
Table 1 (continued)

\begin{tabular}{|c|c|c|c|c|}
\hline Characteristics & $\begin{array}{c}\text { Total } \\
(n=371)\end{array}$ & \multicolumn{2}{|c|}{ The COUNT score } & $P$ value \\
\hline Lymph node metastasis & & & & 0.617 \\
\hline Negative & 342 & 184 & 158 & \\
\hline Positive & 29 & 17 & 12 & \\
\hline 0-I & 117 & 59 & 58 & \\
\hline II & 115 & 70 & 45 & \\
\hline III & 107 & 58 & 49 & \\
\hline IV & 32 & 14 & 18 & \\
\hline The others & 149 & 75 & 74 & \\
\hline $\begin{array}{l}\text { Complications } \\
\text { (Clavien-Dindo } \geq I I)\end{array}$ & & & & 0.927 \\
\hline Absent & 154 & 83 & 71 & \\
\hline Present & 217 & 118 & 99 & \\
\hline Hospital stays (day) & & & & $0.046^{\star}$ \\
\hline Median [IQR] & 21 [16-29] & 20 [15-28] & 22 [16-32] & \\
\hline
\end{tabular}

*, statistically significant $(\mathrm{P}<0.05)$. DM, diabetes mellitus; CA19-9, carbohydrate antigen 19-9; differ, differentiation of tumor cell; AJCC7, American Joint Committee on Cancer seventh edition.

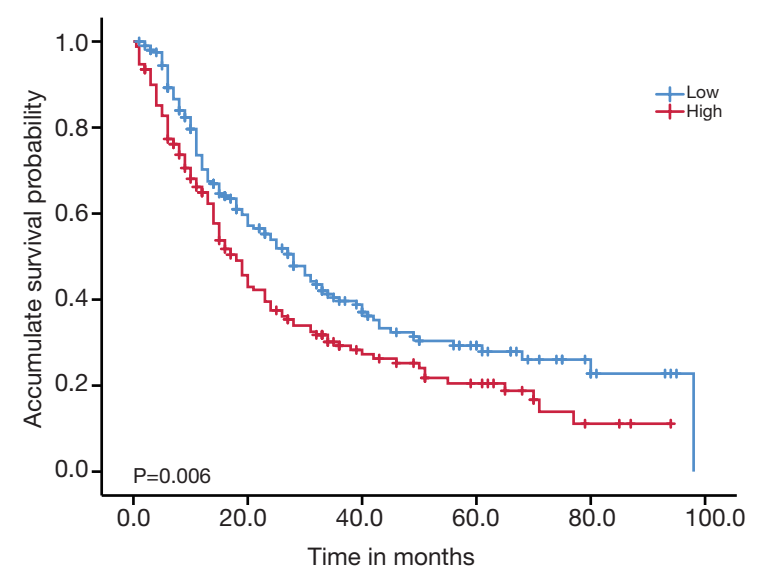

Figure 1 Kaplan-Meier survival analysis of the correlation between the CONUT score and OS among the entire patient cohort. COUNT, controlling nutritional status; OS, overall survival. associated with a poor prognosis (log-rank $\mathrm{P}=0.006)$ (Figure 1).

In addition, univariate and multivariate analyses in the development cohort showed that the CONUT score was an independent predictor of OS [hazard ratio (HR) $1.478,95 \%$ confidence interval (CI) 1.078-2.025, $\mathrm{P}=0.015$ ] (Table 2). In univariate analysis, basic characteristics, basic diseases and symptoms, preoperative biomarkers, surgical method (curative resection with $\mathrm{R} 0$ resection or not), and TNM stage were included. Only CA19-9, CONUT score, surgical method, and TNM stage were significantly associated with OS and were included in the multivariate analysis. In addition to the CONUT score, CA19-9 (HR 2.078, 95\% CI, 1.385-3.119, $\mathrm{P}<0.001)$, surgical method (HR 2.282, 95\% CI, 1.645-3.165, $\mathrm{P}<0.001$ ), and AJCC TNM stage (HR 1.993, 95\% CI, 1.299-3.057, $\mathrm{P}=0.002$ and 
Table 2 Univariate and multivariate cox hazards analysis between clinicopathological features and OS in the development cohort

\begin{tabular}{|c|c|c|c|c|}
\hline Variable & \multicolumn{2}{|c|}{ Univariate analysis } & \multicolumn{2}{|c|}{ Multivariate analysis } \\
\hline \multicolumn{5}{|l|}{ Age (years) } \\
\hline$\geq 65$ vs. $<65$ & $1.060(0.776-1.446)$ & 0.716 & - & - \\
\hline \multicolumn{5}{|l|}{ Sex } \\
\hline \multicolumn{5}{|l|}{ DM } \\
\hline Present vs. absent & $1.264(0.884-1.809)$ & 0.199 & - & - \\
\hline \multicolumn{5}{|l|}{ Hypertension } \\
\hline Present vs. absent & $0.824(0.592-1.147)$ & 0.251 & - & - \\
\hline \multicolumn{5}{|l|}{ CA19-9 (U/mL) } \\
\hline$\geq 40$ vs. $<40$ & $2.368(1.591-3.524)$ & $<0.001^{*}$ & $2.078(1.385-3.119)$ & $<0.001^{*}$ \\
\hline \multicolumn{5}{|l|}{ COUNT } \\
\hline$\geq 2$ vs. $\leq 1$ & $1.431(1.050-1.949)$ & $0.023^{*}$ & $1.478(1.078-2.025)$ & $0.015^{\star}$ \\
\hline \multicolumn{5}{|l|}{ Surgical method } \\
\hline The others $v s$. radical and $\mathrm{R} 0$ resection & $2.549(1.867-3.480)$ & $<0.001^{*}$ & $2.282(1.645-3.165)$ & $<0.001^{*}$ \\
\hline TNM stage (AJCC7) & & $<0.001^{*}$ & & $<0.001^{*}$ \\
\hline $0-1$ & Reference & & Reference & \\
\hline
\end{tabular}

*, statistically significant $(\mathrm{P}<0.05)$. OS, overall survival; $95 \% \mathrm{Cl}, 95 \%$ confidence interval; HR, hazard ratio; DM, diabetes mellitus; CA19-9, carbohydrate antigen 19-9; COUNT, controlling nutritional status; AJCC7, American Joint Committee on Cancer seventh edition.

HR 2.418, 95\% CI, 1.648-3.547, $\mathrm{P}<0.001$, respectively, for stage II and stage III-IV) were found to be independent risk factors for the prognosis of BTCs and were used in the final nomogram model.

\section{Development and validation of the nomogram}

All patients were randomly divided into two cohorts: $263(70.9 \%)$ in the development cohort and the other $108(29.1 \%)$ in the validation cohort. The detailed clinicopathological characteristics are shown in Table S2. There were no significant differences between the two groups. Based on the development cohort, a nomogram was generated to predict 1-, 3-, and 5-year OS in patients with BTC incorporating the four independent prognostic factors
(CONUT, CA19-9, surgical method, and AJCC TNM stage) (Figure 2).

In the development cohort, the C-index was 0.714 (95\% CI, 0.673-0.755) in the nomogram, which was higher than that in the AJCC TNM staging system $(0.589,95 \%$ CI, $0.544-0.634)$, and the AUC of 1-, 3-, and 5-year OS in the nomogram was also higher than that in the AJCC TNM staging system $(0.738,0.781,0.823$ vs. $0.593,0.642,0.701$, respectively) (Table 3). In the validation cohort, the $\mathrm{C}$-index was 0.679 (95\% CI, 0.616-0.742) in the nomogram and 0.592 (95\% CI, 0.527-0.657) in the AJCC TNM staging system (Table 3). The AUCs for 1-, 3-, and 5-year OS were $0.721,0.738$, and 0.816 , respectively, in the nomogram, and $0.592,0.624$, and 0.647 , respectively, in the AJCC TNM staging system. 


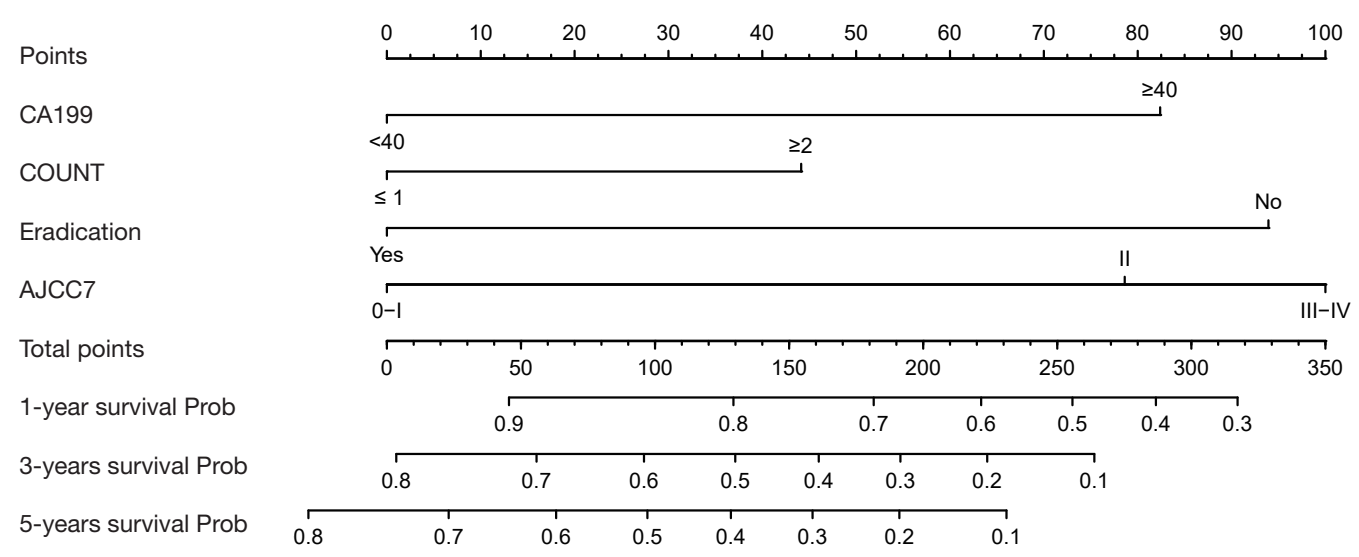

Figure 2 Nomogram to predict the probability of 1-, 3-, and 5-year overall survival (OS) including the CA19-9 level, the CONUT score, the surgical method (Eradication), and the AJCC7 TNM stage in BTC patients. The nomogram can be used to obtain the probability of 1-, 3-, and 5-year OS by adding up the points identified on the point scale for each variable. CA19-9, carbohydrate antigen 19-9; COUNT, controlling nutritional status; Eradication, curative resection with clear margins (R0 resection); AJCC7, American Joint Committee on Cancer seventh edition; Prob, probability.

Table 3 C-index and AUC of the nomogram and AJCC TNM stage in the development and validation cohorts

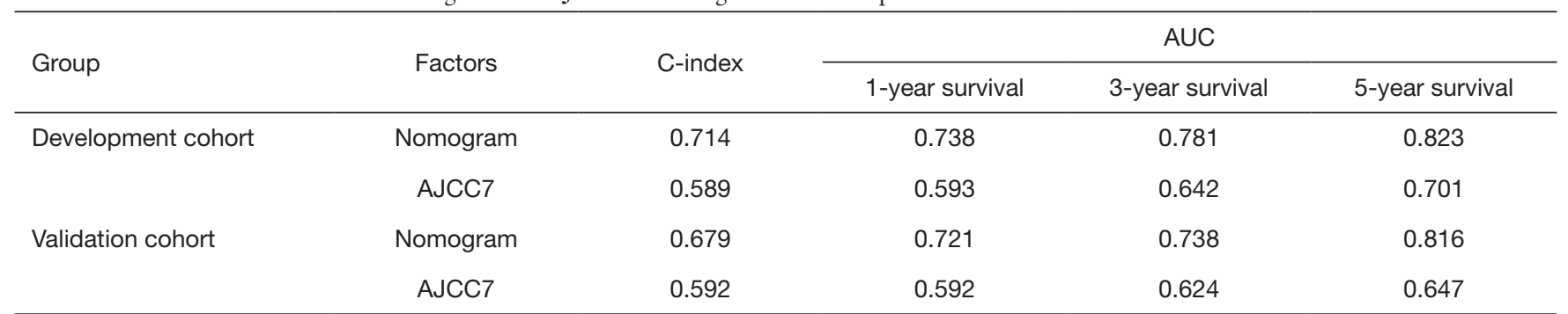

C-index, Harrell's C-index; AUC, area under the curve; AJCC7, American Joint Committee on Cancer seventh edition.

Figure $3 A, B, C, D$ show an intuitively higher AUC in the nomogram than that in the AJCC TNM staging system throughout the first 60 months in the development cohort. And Figure 3E,F,G,H show the similar superiority for AUC of nomogram in the validation cohort. The calibration plot for OS probability at 1,3 , and 5 years after surgery shows a high consistency between the prediction made using the nomogram and the actual survival observations in the development cohort (Figure 4A,B,C) and in the validation cohort (Figure $4 D, E, F)$. The DCA for the nomogram and single prognostic factors in the development cohort is shown in Figure $5 A, B, C$. It shows that the nomogram for predicting OS was more useful than the none or all patients deceased scheme under a wide range of thresholds $(>20 \%$, $>40 \%$, and $>50 \%$ for $1-, 3$-, and 5-year OS, respectively). Furthermore, the clinical net benefit of the nomogram was better than all other single prognostic factors. And in the validation cohort, the DCA for the nomogram and the single prognostic factors is shown in Figure 5D,E,F. The nomogram for predicting OS still shows a comparable clinical net benefit.

\section{Risk stratification of OS using the nomogram}

According to the nomogram generated in this study, all cases were divided into four risk groups, which showed good prognostic classification of patients with BTC in the development cohort. There were 86, 95, 95, and 95 cases in the low-, medium-, high-, and very high-risk groups, respectively, and the mean OS was $67.39 \pm 8.75,40.78 \pm 8.01$, $26.84 \pm 5.13$, and $14.71 \pm 2.83$ months $(\mathrm{P}<0.001)$, respectively, using the nomogram model (Figure $6 A$ ), compared with 

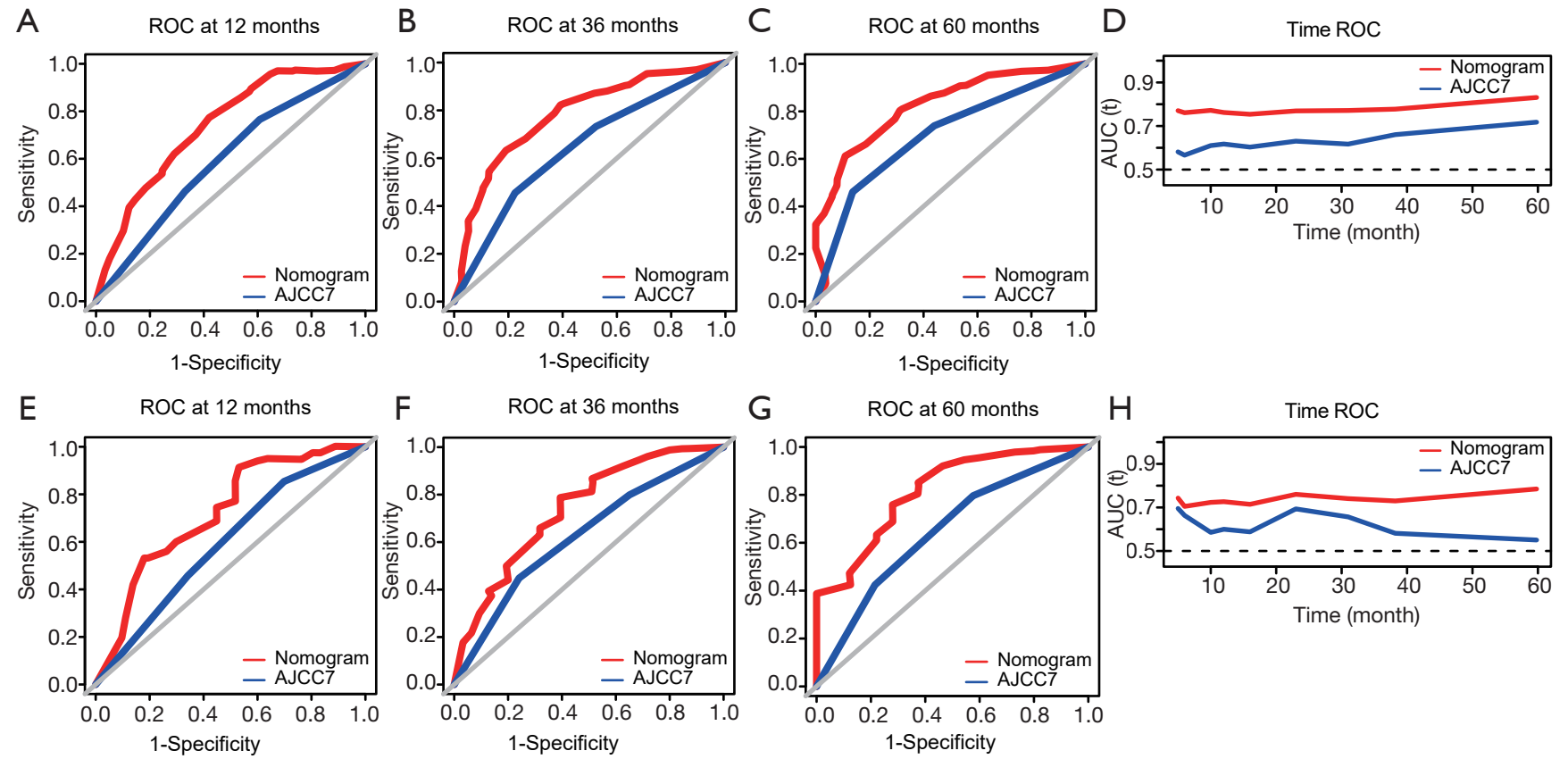

Figure 3 ROC curve and time ROC curve analyses to compare the predictive performance. (A,B,C) ROC curve analyses of 1-year (A), 3-year (B) and 5-year (C) OS in the development cohort. (D) Time ROC curve analyses in the development cohort. (E,F,G) ROC curve analyses of 1-year (E), 3-year (F) and 5-year (G) OS in the validation cohort. (H) Time ROC curve analyses in the validation cohort. ROC, Receiver operating characteristic; AJCC7, American Joint Committee on Cancer seventh edition; AUC, area under the curves.

$50.51 \pm 7.92,35.69 \pm 6.74,28.66 \pm 5.93$, and $12.79 \pm 5.73$ months $(\mathrm{P}<0.001)$, respectively, based on the AJCC TNM staging system (Figure 6B). Furthermore, the effect of the risk stratification of our nomogram model in the three subgroups of different cancers was tested, and a good prognostic classification was presented for GBC, ICC, and ECC patients (all $\mathrm{P}<0.001$ ) (Figure 6C,D,E). In addition, the subgroups for the different surgical methods were also tested, and our risk stratification still showed satisfactory prognostic classification $(\mathrm{P}<0.001$ and $\mathrm{P}=0.007$, respectively) (Figure $6 F, G$ ). However, in most of the subgroups, the AJCC TNM staging system could not discriminate between the stage II and stage III groups (Figure 6H,I,7,K,L). For stage II and stage III, mean OS rates were $31.0 \pm 6.22$ and $33.72 \pm 9.48$ months, respectively, in ECC patients, and $44.86 \pm 8.55$ and $37.58 \pm 9.32$ months, respectively, in the complete resection group. In our nomogram model, the medium-risk and high-risk groups were discriminated well, and the mean OS rates were 44.26 49.56 and $28.44 \pm 7.47$ months, respectively, in ECC patients and $40.96 \pm 8.09$ and $29.37 \pm 6.60$ months, respectively, in the complete resection group.

\section{Discussion}

BTCs are relatively rare but deadly cancers, and their incidence seems to be increasing globally. Surgery is the most common and potentially curative treatment; however, many patients are not eligible for surgery at the time of diagnosis. In addition to the decreased chance of being eligible for surgery, the prognosis is even worse because of the high rate of recurrence. Although the AJCC TNM staging system is the most widely used staging system, it does not take into account tumor heterogeneity or comprehensively includes the various disease states of patients, even for those in the same TNM stage; thus, patients may have rather different prognoses $(22,23)$. Therefore, we aimed to develop a more accurate prognostic risk stratification system to help guide the individualized choice of surgery and other treatments. In this study, the preoperative CONUT score was found to be an independent prognostic marker for OS in BTCs, and a 


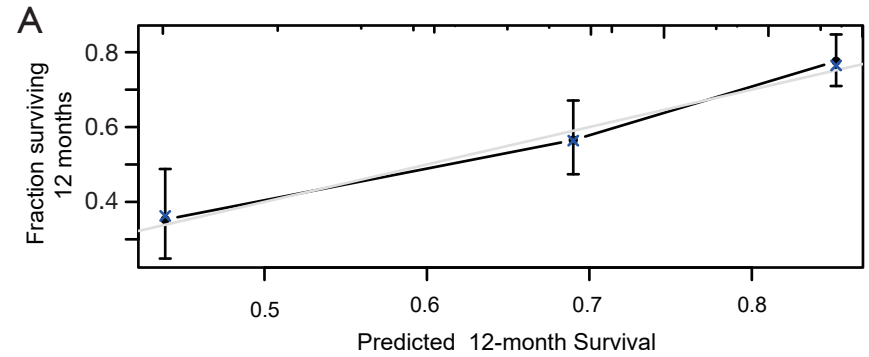

C

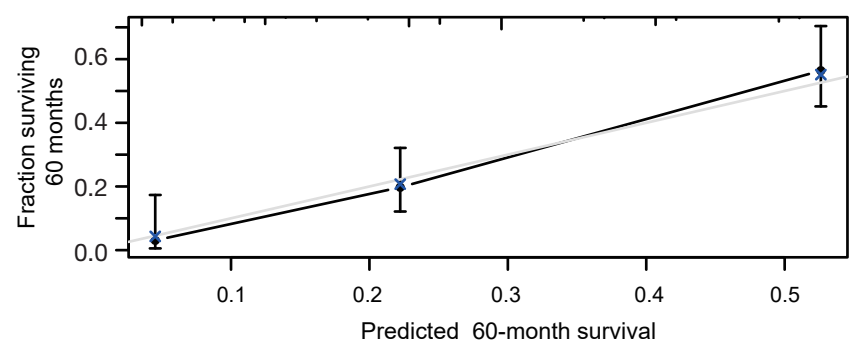

E

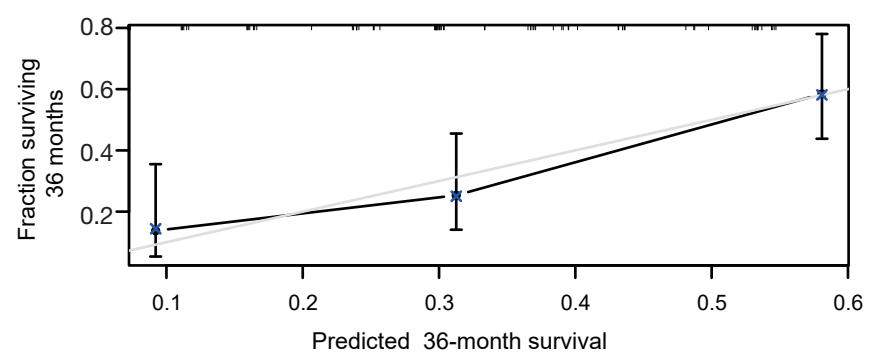

B

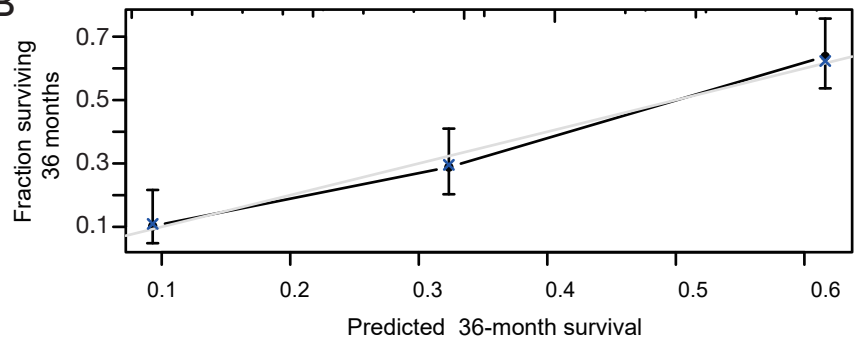

D

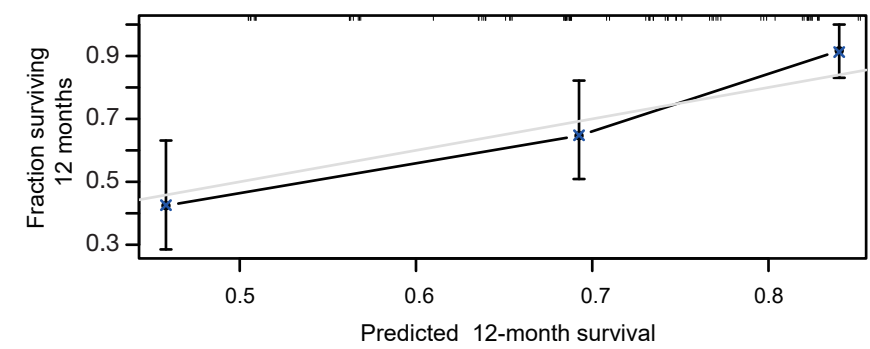

F

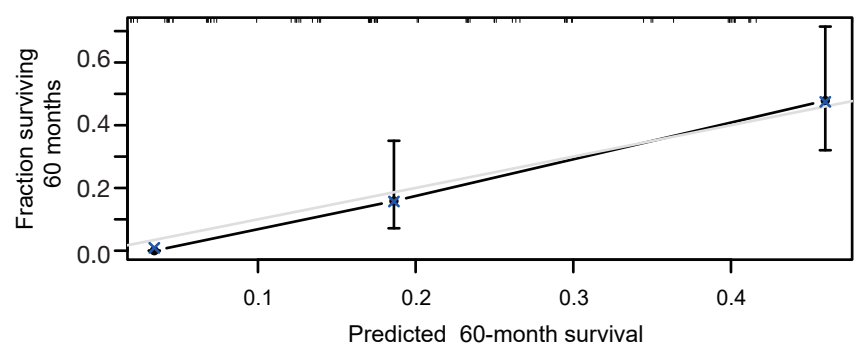

Figure 4 Calibration curve of the nomogram both in the development and validation cohorts. (A,B,C) 1-year (A), 3-year (B) and 5-year (C) OS in the development cohort. (D,E,F) 1-year (D), 3-year (E) and 5-year (F) OS in the validation cohort. OS, overall survival.

well-calibrated prognostic nomogram was established based on CONUT scores and other prognostic factors to predict OS after surgery. Compared with the AJCC TNM staging system, the nomogram had superior prognostic discriminative power and could provide a clinical benefit.

The prognostic significance of the CONUT score has been elucidated in many types of cancer. However, this study was the first to explore the utility of the CONUT score in BTCs. It was found that a high CONUT score was significantly associated with a poor OS and long hospital stays after surgery. Furthermore, the CONUT score was an independent prognostic marker for OS. The CONUT score was calculated using serum albumin and cholesterol levels and lymphocyte count in peripheral blood, and therefore, it could reflect the nutritional condition and systemic inflammatory status of patients. It has been found that tumor progression and treatment tolerance were closely correlated with the nutritional and inflammatory states (10).
Hence, it is understandable that the CONUT score could be used as a prognostic factor in tumor patients. More specifically, each of the three components of the CONUT score-markers of protein stores, lipid metabolism, and immune defenses-reflect cancer progression (24). Ellegård and Bosaeus concluded that the systemic inflammatory response was the most important biochemical index in malignant disease (25). Serum albumin concentrations could be lower because of the systemic inflammatory response in cancer patients, and therefore, it is primarily a marker of inflammation instead of nutrition when considering its prognostic importance in cancer patients (26). Total cholesterol concentration has been reported to correlate with tumor progression and prognosis because tumor tissues reduce plasma cholesterol concentrations and caloric intake (27). As for lymphocytes, they are considered to initiate cytotoxic immune responses by inducing cell apoptosis and suppressing tumor cell proliferation, invasion, 
A

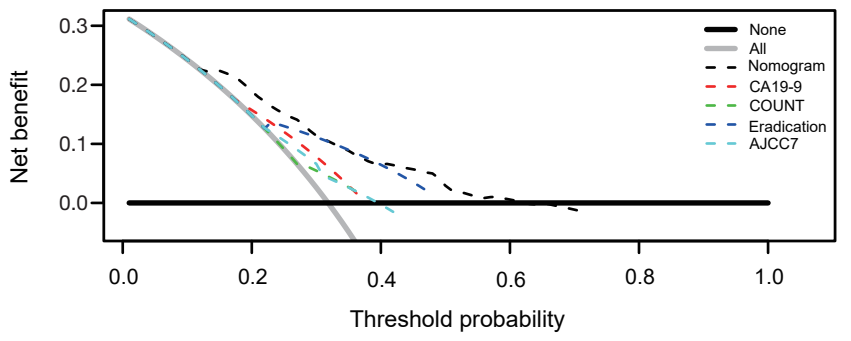

C

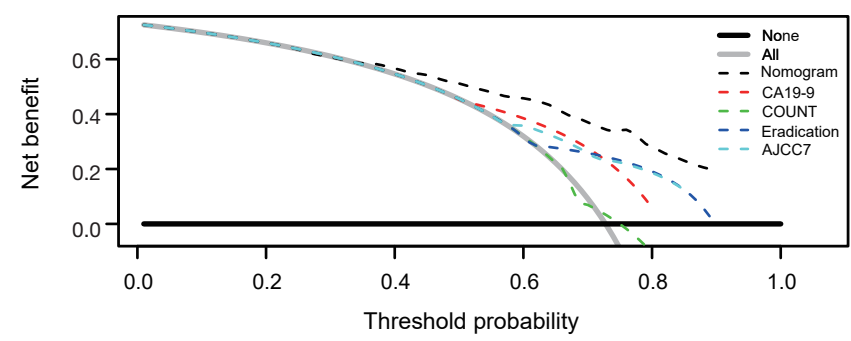

$\mathrm{E}$

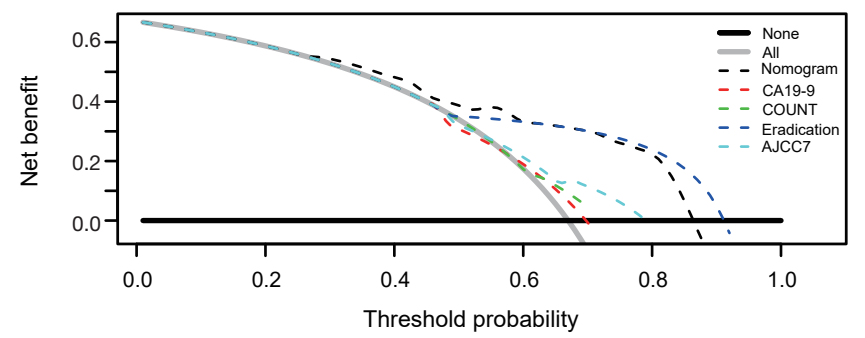

B

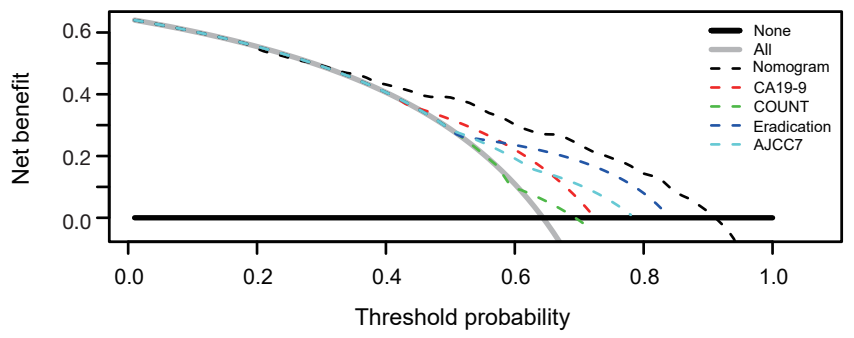

D

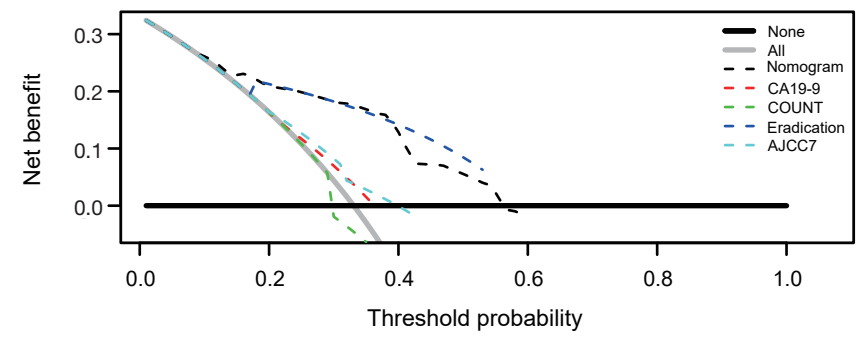

$\mathrm{F}$

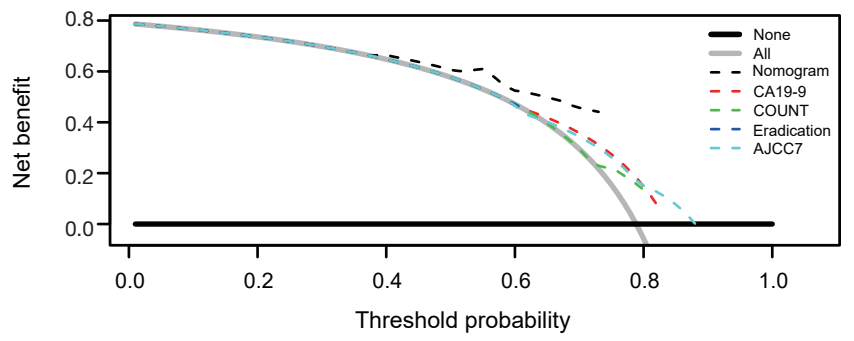

Figure 5 Decision curve analysis for OS. (A,B,C) 1-year (A), 3-year (B) and 5-year (C) OS in the development cohort. (D,E,F) 1-year (D), 3-year (E) and 5-year (F) OS in the validation cohort. Black line: all patients died. Gray line: no patients died. Dashed black line: nomogram model. Dashed red line: CA19-9 staging system model. Dashed green line: CONUT staging system model. Dashed dark blue line: surgical method staging system model. Dashed blue line: AJCC TNM staging system model. CA19-9, carbohydrate antigen 19-9; COUNT, controlling nutritional status; Eradication, curative resection with clear margins (R0 resection); AJCC7, American Joint Committee on Cancer seventh edition.

and migration (28). In addition, the three parameters are easy to obtain in a blood examination. In summary, the CONUT score is of great value to help determine the prognosis of patients with BTC.

As the most frequently used tool to evaluate prognosis, the AJCC TNM staging system does not have enough predictive power to provide individualized assessment of survival. It only contains anatomic information, but there are other important prognostic factors, such as nutritional and inflammatory states as well as tumor biomarkers, to determine the heterogeneity of patients. Furthermore, the curative effect of surgery could affect a patient's prognosis. In our study, according to multivariate analysis, these factors were considered and included in the final nomogram: the level of serum CA19-9, CONUT score, surgical method, and AJCC TNM stage. Taking the tumor biomarker, nutritional/inflammatory state, tumor stage, and surgical method together, the nomogram contained more comprehensive information and was more accurate in predicting OS in patients with BTC than the AJCC TNM staging system. More precisely, our nomogram had higher $\mathrm{C}$ indexes and AUC values than the AJCC TNM staging system, which meant that it could better discriminate between patients. Additionally, given that our nomogram was well calibrated, the clinical prognostic value was better than any single prognostic factor. 
A

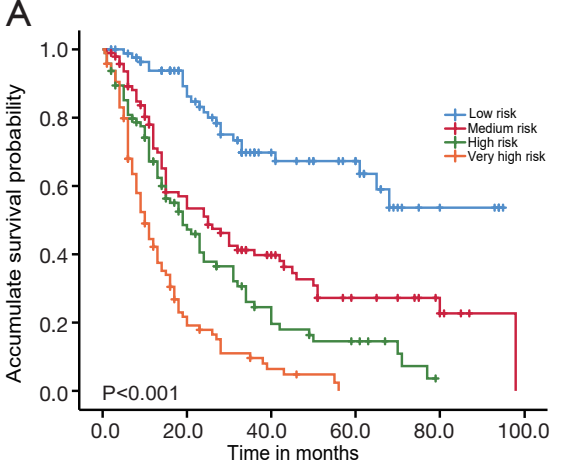

D

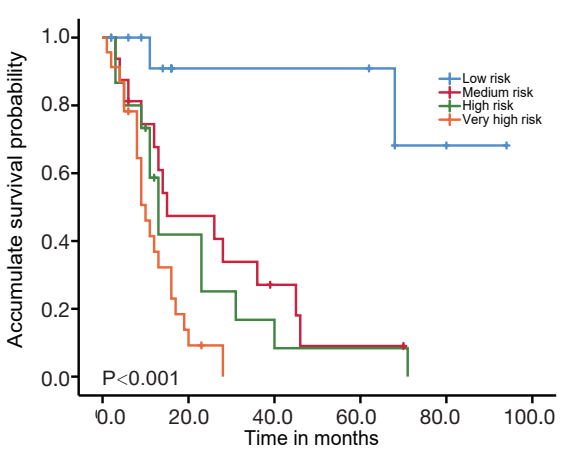

G

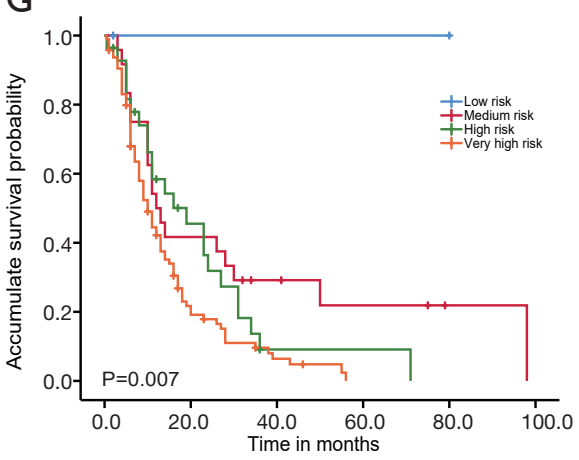

J

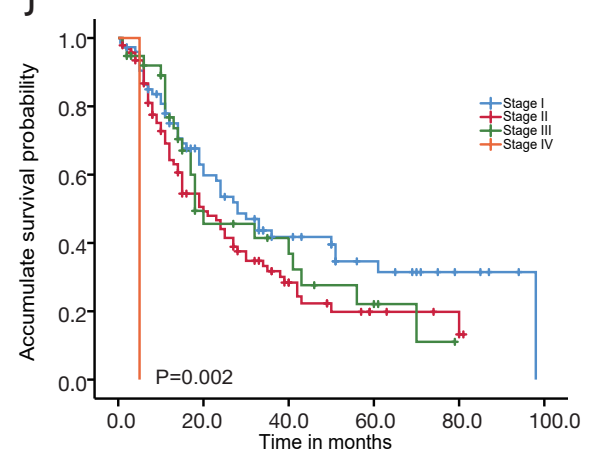

B

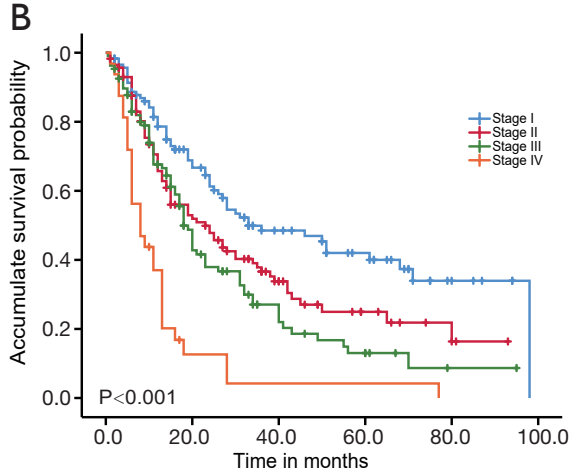

E

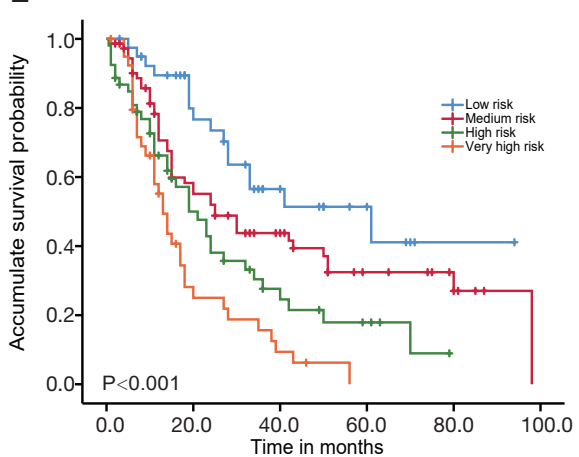

$\mathrm{H}$

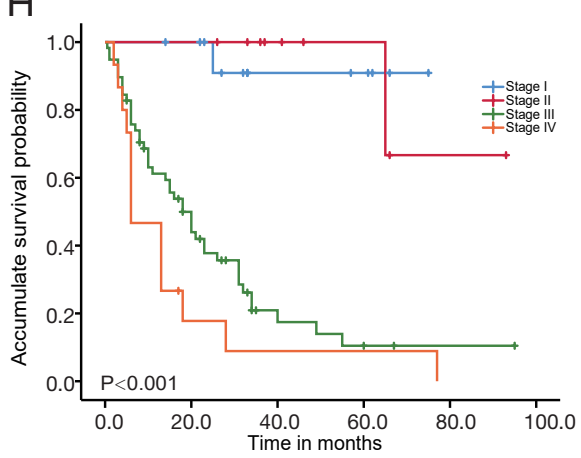

$\mathrm{K}$

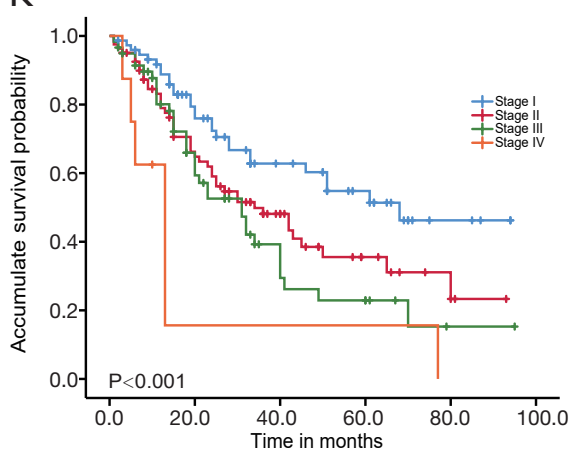

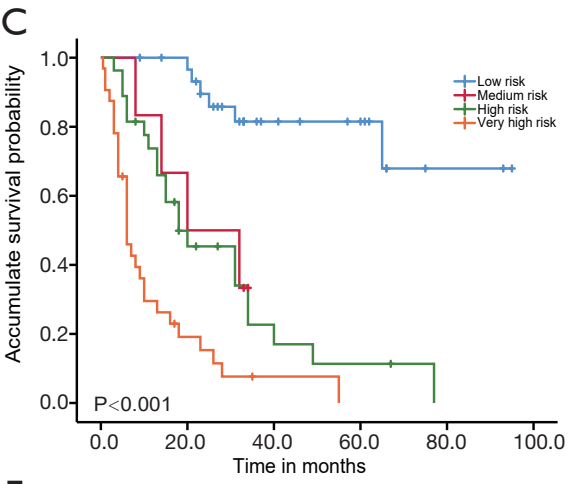

$\mathrm{F}$
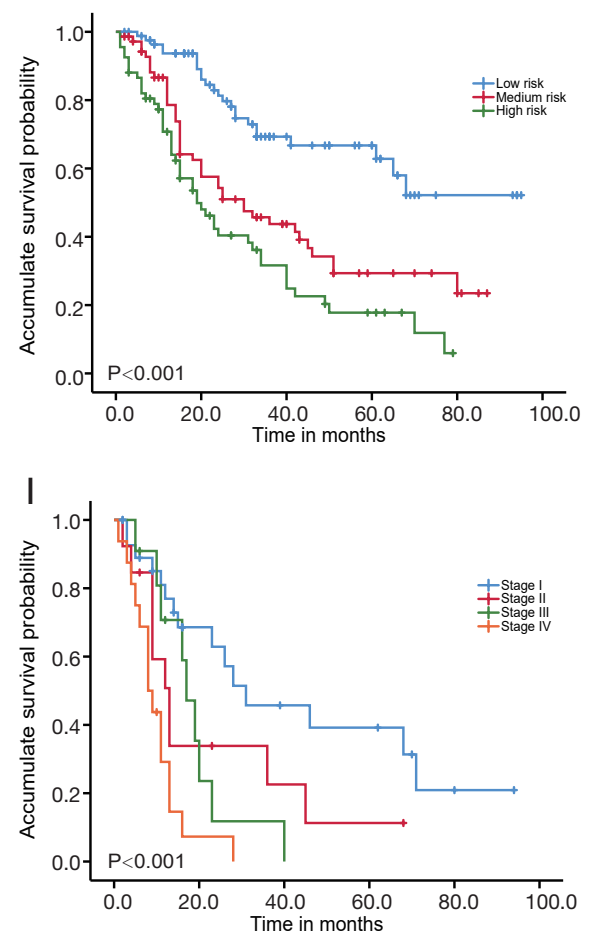

L

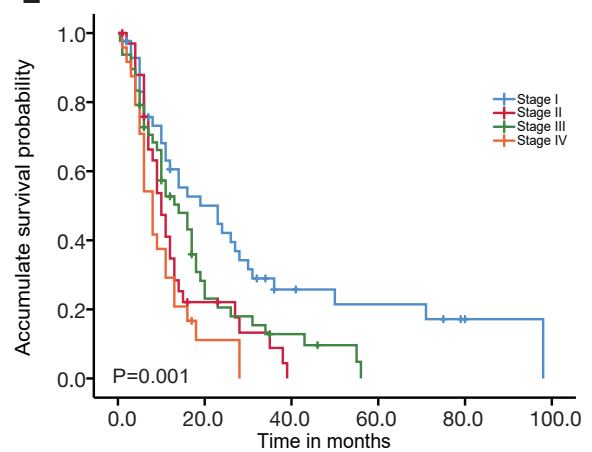

Figure 6 Overall survival in patients with BTCs classified by the nomogram model in the entire cohort (A), the AJCC7 TNM staging system in the entire cohort (B), the nomogram model in GBC (C), ICC (D), and ECC (E) patients, the nomogram model in the complete resection (F) and the other group (G), AJCC7 TNM staging system in GBC (H), ICC (I), and ECC (J) patients, and the AJCC7 TNM staging system in the complete resection $(\mathrm{K})$ and not complete resection group (L). BTCs, biliary tract cancers; AJCC7, American Joint Committee on Cancer seventh edition; GBC, gallbladder cancer; ICC, intrahepatic cholangiocarcinomas; ECC, extrahepatic cholangiocarcinomas. 
Our nomogram had good risk stratification power for patients with BTC in the entire cohort and in the subgroups. The results showed that the nomogram could reliably discriminate among patients at different levels of risk in every BTC subgroup, including GBCs, ICCs, and ECCs, indicating that our prognostic model could be applied to patients with the three different types of BTC. Similarly, the model was tested in patients who received curative resection with clear margins and non-radical surgery, and it could also discriminate reliably. Therefore, the nomogram could be used to predict OS in patients undergoing different surgical procedures. Furthermore, our nomogram was practical, utilizing accessible clinical information and returning intuitive predictive results of 1-, 3-, and 5-year OS. In summary, for patients with BTC who will undergo complete resection or other types of surgery, our nomogram, which utilizes accessible preoperative biomarkers and the tumor TNM stage, could provide an accurate prediction of OS and thus, be a useful tool for patient counseling and personalized treatment management.

This study had several limitations. First, the nomogram was generated based on data from a single institution in China, and a large percentage of cases with missing data were excluded, which might have introduced some selection bias. Given the heterogeneity of the population, more samples from multiple centers should be included in validating our nomogram. Second, the study was conducted retrospectively, which made it susceptible to the inherent biases of such a study format. Finally, we only evaluated OS in our study, and cancer-specific survival (CSS) or recurrence-free survival (RFS) should be evaluated in future research.

\section{Conclusions}

Our study is the first to demonstrate that the CONUT score is an independent prognostic marker for OS after surgery in patients with BTCs. Based on the CONUT score, CA19-9, surgical method, and AJCC TNM stage, the nomogram could more accurately predict OS than the AJCC TNM staging system alone and thus provide more individualized guidance regarding treatment choice.

\section{Acknowledgments}

Funding: We acknowledge the CAMS Innovation Fund for Medical Sciences (CIFMS) (No.2016-I2M-1-001) and Tsinghua University-Peking Union Medical College
Hospital Cooperation Project (PTQH201904552).

\section{Footnote}

Reporting Checklist: The authors have completed the STROBE reporting checklist. Available at http://dx.doi. org/10.21037/atm-20-6770

Data Sharing Statement: Available at http://dx.doi. org/10.21037/atm-20-6770

Peer Review File: Available at http://dx.doi.org/10.21037/ atm-20-6770

Conflicts of Interest: All authors have completed the ICMJE uniform disclosure form (available at http://dx.doi. org/10.21037/atm-20-6770). The authors have no conflicts of interest to declare.

Ethical Statement: The authors are accountable for all aspects of the work in ensuring that questions related to the accuracy or integrity of any part of the work are appropriately investigated and resolved. The study was approved by the Clinical Research Ethics Committee of Peking Union Medical College Hospital (approval No. S-K1110). Informed consent forms were signed by all patients before surgery to permit the use of their clinical data in the study. All procedures performed in studies involving human participants were in accordance with the 1964 Helsinki Declaration and its later amendments or comparable ethical standards.

Open Access Statement: This is an Open Access article distributed in accordance with the Creative Commons Attribution-NonCommercial-NoDerivs 4.0 International License (CC BY-NC-ND 4.0), which permits the noncommercial replication and distribution of the article with the strict proviso that no changes or edits are made and the original work is properly cited (including links to both the formal publication through the relevant DOI and the license). See: https://creativecommons.org/licenses/by-nc-nd/4.0/.

\section{References}

1. Valle JW, Lamarca A, Goyal L, et al. New Horizons for Precision Medicine in Biliary Tract Cancers. Cancer Discov 2017;7:943-62.

2. Miranda-Filho A, Pineros M, Ferreccio C, et al. 
Gallbladder and extrahepatic bile duct cancers in the Americas: Incidence and mortality patterns and trends. Int J Cancer 2020;147:978-89.

3. Siegel RL, Miller KD, Jemal A. Cancer statistics, 2019. CA Cancer J Clin 2019;69:7-34.

4. Zhang $\mathrm{H}$, Yang $\mathrm{T}, \mathrm{Wu} \mathrm{M}$, et al. Intrahepatic cholangiocarcinoma: Epidemiology, risk factors, diagnosis and surgical management. Cancer Lett 2016;379:198-205.

5. Raoof $M$, Singh G. Rising trends in intrahepatic cholangiocarcinoma incidence and mortality: getting at the root cause. Hepatobiliary Surg Nutr 2019;8:301-3.

6. Gorgen A, Goldaracena N, Zhang W, et al. Intrahepatic cholangiocarcinoma, are we making progress? Hepatobiliary Surg Nutr 2018;7:127-9.

7. Doussot A, Jarnagin WR, Azoulay D, et al. Improving actual survival after hepatectomy for intrahepatic cholangiocarcinoma-still a long way to go. Hepatobiliary Surg Nutr 2019;8:161-3.

8. Mao M, Zhang A, He Y, et al. Development and validation of a novel nomogram to predict overall survival in gastric cancer with lymph node metastasis. Int J Biol Sci 2020;16:1230-7.

9. Groot Koerkamp B, Fong Y. Outcomes in biliary malignancy. J Surg Oncol 2014;110:585-91.

10. Tsilimigras DI, Mehta R, Aldrighetti L, et al. Development and Validation of a Laboratory Risk Score (LabScore) to Predict Outcomes after Resection for Intrahepatic Cholangiocarcinoma. J Am Coll Surg 2020;230:381-391.e2.

11. Tsilimigras DI, Moris D, Mehta R, et al. The systemic immune-inflammation index predicts prognosis in intrahepatic cholangiocarcinoma: an international multiinstitutional analysis. HPB (Oxford) 2020;22:1667-74.

12. Zhang Y, Shi SM, Yang H, et al. Systemic inflammation score predicts survival in patients with intrahepatic cholangiocarcinoma undergoing curative resection. J Cancer 2019;10:494-503.

13. Zhou H, Chao W, Cui L, et al. Controlling Nutritional Status (CONUT) score as immune-nutritional predictor of outcomes in patients undergoing peritoneal dialysis. Clin Nutr 2020;39:2564-70.

14. Suzuki S, Kanaji S, Yamamoto M, et al. Controlling Nutritional Status (CONUT) Score Predicts Outcomes of Curative Resection for Gastric Cancer in the Elderly. World J Surg 2019;43:1076-84.

15. Qiu Y, You J, Wang K, et al. Effect of whole-course nutrition management on patients with esophageal cancer undergoing concurrent chemoradiotherapy: A randomized control trial. Nutrition 2020;69:110558.

16. Yang C, Wei C, Wang S, et al. Combined Features Based on Preoperative Controlling Nutritional Status Score and Circulating Tumour Cell Status Predict Prognosis for Colorectal Cancer Patients Treated with Curative Resection. Int J Biol Sci 2019;15:1325-35.

17. Harimoto N, Yoshizumi T, Inokuchi S, et al. Prognostic Significance of Preoperative Controlling Nutritional Status (CONUT) Score in Patients Undergoing Hepatic Resection for Hepatocellular Carcinoma: A Multiinstitutional Study. Ann Surg Oncol 2018;25:3316-23.

18. Miyata T, Yamashita YI, Higashi T, et al. The Prognostic Impact of Controlling Nutritional Status (CONUT) in Intrahepatic Cholangiocarcinoma Following Curative Hepatectomy: A Retrospective Single Institution Study. World J Surg 2018;42:1085-91.

19. Shoji F, Haratake N, Akamine T, et al. The Preoperative Controlling Nutritional Status Score Predicts Survival After Curative Surgery in Patients with Pathological Stage I Non-small Cell Lung Cancer. Anticancer Res 2017;37:741-7.

20. Li W, Li M, Wang T, et al. Controlling Nutritional Status (CONUT) score is a prognostic factor in patients with resected breast cancer. Sci Rep 2020;10:6633.

21. Xu H, Tan P, Jin X, et al. Validation of the preoperative controlling nutritional status score as an independent predictor in a large Chinese cohort of patients with upper tract urothelial carcinoma. Cancer Med 2018;7:6112-23.

22. He C, Cai Z, Zhang Y, et al. Prognostic Model to Predict Cancer-Specific Survival for Patients With Gallbladder Carcinoma After Surgery: A Population-Based Analysis. Front Oncol 2019;9:1329.

23. Mahar AL, Zagorski B, Kagedan D, et al. Evaluating TNM stage prognostic ability in a population-based cohort of gastric adenocarcinoma patients in a lowincidence country. Can J Public Health 2018;109:480-8.

24. Ryo S, Kanda M, Ito S, et al. The Controlling Nutritional Status Score Serves as a Predictor of Short- and LongTerm Outcomes for Patients with Stage 2 or 3 Gastric Cancer: Analysis of a Multi-institutional Data Set. Ann Surg Oncol 2019;26:456-64.

25. Ellegård LH, Bosaeus IG. Biochemical indices to evaluate nutritional support for malignant disease. Clin Chim Acta 2008;390:23-7.

26. Crumley AB, Stuart RC, McKernan M, et al. Is hypoalbuminemia an independent prognostic factor in patients with gastric cancer? World J Surg 2010;34:2393-8. 


\section{Page 14 of 14}

27. Iseki Y, Shibutani M, Maeda K, et al. Impact of the Preoperative Controlling Nutritional Status (CONUT) Score on the Survival after Curative Surgery for Colorectal Cancer. PLoS One 2015;10:e0132488.

28. Liu X, Zhang D, Lin E, et al. Preoperative controlling

Cite this article as: Sun L, Su S, Xiong J, Hu W, Liu L, Xu H, Du S, Zhao H, Lu X, Sang X, Zhong S, Yang H, Mao Y. Controlling nutritional status score as a prognostic marker to predict overall survival in resected biliary tract cancers. Ann Transl Med 2021;9(8):644. doi: 10.21037/atm-20-6770

\section{Sun et al. The prognostic value of CONUT in resected BTC}

nutritional status (CONUT) score as a predictor of longterm outcome after curative resection followed by adjuvant chemotherapy in stage II-III gastric Cancer. BMC Cancer 2018;18:699. 
Supplementary

Table $\mathbf{S 1}$ The CONUT scoring system

\begin{tabular}{|c|c|c|c|c|}
\hline Parameters & Normal & Light & Moderate & Severe \\
\hline Score & 0 & 2 & 4 & 6 \\
\hline Total lymphocyte count $\left(/ \mathrm{mm}^{3}\right)$ & $\geq 1,600$ & $1,200-1,599$ & $800-1,199$ & $<800$ \\
\hline Score & 0 & 1 & 2 & 3 \\
\hline Score & 0 & 1 & 2 & 3 \\
\hline CONUT score (total) & $0-1$ & $2-4$ & $5-8$ & $9-12$ \\
\hline
\end{tabular}

COUNT, controlling nutritional status. 
Table S2 Baseline clinicopathological features in the two cohorts

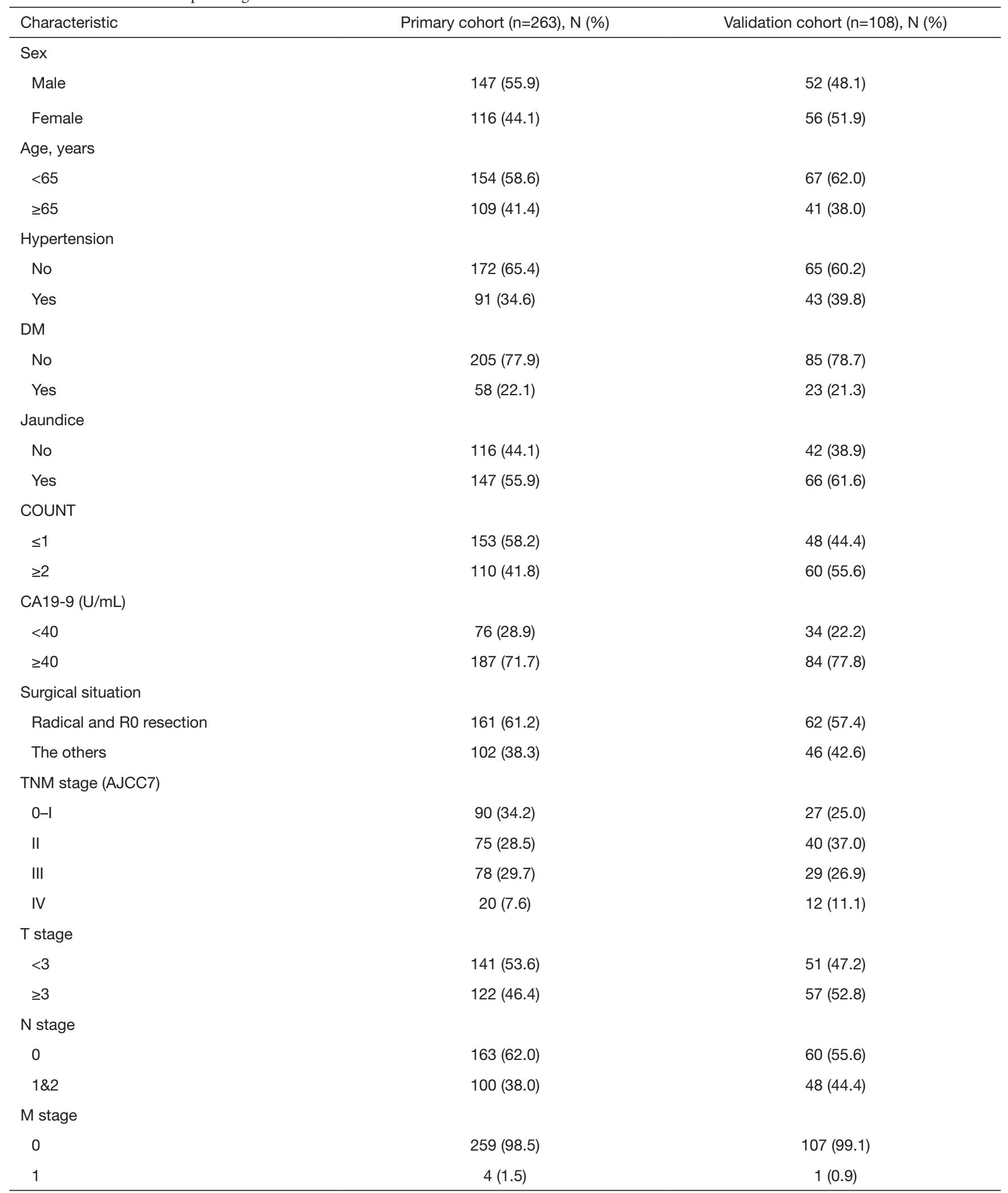

DM, diabetes mellitus; COUNT, controlling nutritional status; CA19-9, carbohydrate antigen 19-9; AJCC7, American Joint Committee on Cancer seventh edition.

(c) Annals of Translational Medicine. All rights reserved. 